\title{
Extraction of gist without encoding of individual items in RSVP of numerical sequences
}

\author{
David Rosenbaum ${ }^{1}$, Howard Bowman ${ }^{2,3}$ \& Marius Usher ${ }^{1}$ \\ ${ }^{1}$ School of Psychological Sciences, Tel-Aviv University, Israel \\ 2 University of Kent at Canterbury, UK \\ ${ }^{3}$ School of Psychology, University of Birmingham, Birmingham B15 2TT, UK
}

\begin{abstract}
We examine the ability of human observers to estimate the average of rapid serial visual presentational (RSVP) sequences of numerical symbols at rates of up to 30 items/sec. In three experiments ( 2 pilots and 1 pre-registered replication), we find that observers extract the average of fast RSVP sequences (which they "barely see") with a precision that exceeds chance and also exceeds what could be achieved based on a (offline) calculation from numbers that are encoded in working-memory at the end of the sequence. The results support a mechanism that extracts the average online and automatically, based on population coding of numerosity detectors and shows a type of gist extraction that takes place without robust access to the individual elements.
\end{abstract}




\section{Introduction}

Recent research has indicated that humans have an impressive ability to extract summary statistics from sets of elements that are briefly presented as spatial arrays or as rapid temporal sequences (Ariely, 2001; Brezis, Bronfman \& Usher, 2015, 2017; Chong \& Treisman, 2003, 2005; Dakin, 2001; Haberman, Harp \& Whitney, 2009; Haberman \& Whittey, 2011; Khayat \& Hochstein, 2018; Parkes, Lundt, Angelucci, Solomon \& Morgan, 2001; Robitaiile \& Haris, 2011; Rosenbaum, Glickman \& Usher, 2020). For example, in a seminal study, Ariely (2001) has shown that participants are sensitive to an average visual property (the mean size) of a set of elements in a visual array, and that this sensitivity does not decrease with the set-size of the array, while at the same time the participants were at chance at identifying if a particular test-probe was presented in the array. Similar results were reported for other types of visual properties, such as emotional expressions (Haberman, Harp \& Whitney, 2009; Haberman \& Whitney, 2011), and (symbolic) numerals (Lee et al, 2021; Rosenbaum, de Gardelle \& Usher, 2021). Moreover, this process appears to take place automatically (or at least without intention), as it appears to take place when the task does not require averaging (Khayat \& Hochstein, 2018).

These results have been interpreted as indicating a gist mode of processing that exceeds the capacity limitation of the visual working memory system of about 3-4 items (Luck \& Vogel, 1997). Similar results were obtained with rapid serial visual presentation (RSVP). Brezis et al. (2015; Exp-3), have shown that people can estimate the numerical average of 2 digit numbers, presented at a rate of $10 / \mathrm{sec}$ and that the precision of the estimation increased with the length of the series (see Rosenbaum, Glickman \& Usher, 2020 for estimations of the sequence variance and for a discussion of individual differences in the estimation mechanism) and similar results were obtained for the averaging of non-symbolic numerosity displays (Katzin et al., 2020). This rules out an estimation strategy based on a symbolic calculation of the average from sequence items retained in working memory (which would predict decreasing precision with sequence length; see Fig 2, in Brezis et al., 2015). The ability to extract gist from RSVP sequences is particularly interesting in the context of findings showing that people are poor in recognizing (at the end of an RSVP sequence of rate higher than 10 items/sec) if a particular item was presented as part of that sequence (Potter, 1976). There is further debate as to whether RSVP sequences (at rates of 10-20 items/sec) generate conscious percepts, which are fleeting (Su, Barnard \& Bowman, 2011) - a type of phenomenal experience without robust access to report (Block, 2011; Bronfman, Jacobson \& Usher, 2019) - or they do not generate any conscious percepts at all. The latter position is consistent with one of the dominant theories of consciousness - the neuronal global-workspace model (Dehaene \& Naccache, 2001; Dehaene, Kerszberg \& Changeux, 1998), according to which, processing of stimuli in perceptual brain areas (as is the case for the unreported items in RSVP or in the Sperling task; Sperling, 1960; see also Block, 2011; Kouider, Sackur \& De Gardelle, 2012) is not sufficient for those stimuli to become conscious. 
Rather, the critical feature that triggers consciousness is an attentional selection that boosts neural activation to trigger the ignition of a global frontal-parietal system (Dehaene, Kerszberg \& Changeux, 1998). ${ }^{1}$

The aim of this study is to probe the temporal limits for which the extraction of summary statistics, in particular - the average - is possible for RSVP of numerical sequences and to probe if the underlying mechanism is mediated by a robust access to individual items in visual $\mathrm{WM}^{2}$. We examine presentation rates from 4 to 31 items $/ \mathrm{sec}$ (i.e., $250-32 \mathrm{~ms} /$ item) and we probe both the estimation of the average and the memory of individual items. In particular, we are interested to determine if observers can estimate the average of an RSVP sequence with a precision that exceeds what could be achieved (offline) based on their memory of individual items at the end of the sequence. Such a result would support the view that extraction of gist can be carried out without a robust conscious access of the elements (Van Opstal, de Lange \& Dehaene, 2011) and that the gist extraction is not only a mode of parallel spatial attention that takes place for arrays with spatial information overload (Baek \& Chong, 2020).

\section{Methods \\ Participants}

A total of 30 healthy undergraduate volunteers from the same pool of participants that often participate in psychology experiments at Tel-Aviv University (all with normal or corrected to normal vision) were recruited to participate in three experiments for an equivalent of $\$ 15$, in a psychophysical lab under special COVID19 safety guidelines (testing conditions were restricted so that the experimenter was not present in the same room as the participants, and all equipment was sterilized before the tests). All procedures and experimental protocols were approved by the ethics committee of the Psychology Department of Tel Aviv University (Application 743/12). All experiments were carried out in accordance with the approved guidelines. The first 2 experiments were pilots with $\mathrm{N}=10$ and $\mathrm{N}=5$, respectively and the third experiment $(\mathrm{N}=15)$ was a pre-registered replication (https://osf.io/7cj9g/).

1 For items presented in RSVP (at rates faster than 10/sec), and without a 'target' to match, the attentional selection cannot take place fast enough to trigger the ignition of the neuronal global workspace, which, according to this view, enables access of items to consciousness.

2 We distinguish between a robust access that involves encoding in working memory or access to the global-workspace, and a transient access that may only allow visual experience of the items (Block, 2011; Bronfman, Jacobson \& Usher, 2018). 


\section{Procedure}

In all 3 experiments (see Fig. 1), each trial presented a sequence of 12 1-digit numerals ( 1 to 9 , sampled in each trial from one of the three distributions in Fig $1 \mathrm{C}^{3}$ ), which were followed by a post sequence mask (symbol "\$”). The RSVP presentation rate varied (between blocks) from 4-31 items/sec (corresponding to item-durations of $250 \mathrm{~ms}$ to $50 \mathrm{~ms}$ in Exp. 1 and from $250 \mathrm{~ms}$ to $32 \mathrm{~ms}$, in Exp. 2 \& 3). The blocks were carried out from slow to fast: the $1^{\text {st }}$ block had a presentation duration (per item) of 250ms, followed by blocks with 150ms, 100ms, 50ms (Exp-1) and $32 \mathrm{~ms}$ (in Exp. 2 \& 3 only), in order to enable the participants to tune in with the task before they reach the most difficult condition. At each presentation-rate block, the subjects were required to estimate the sequence's average with a slider on a continuous scale (right in Fig. 1A).

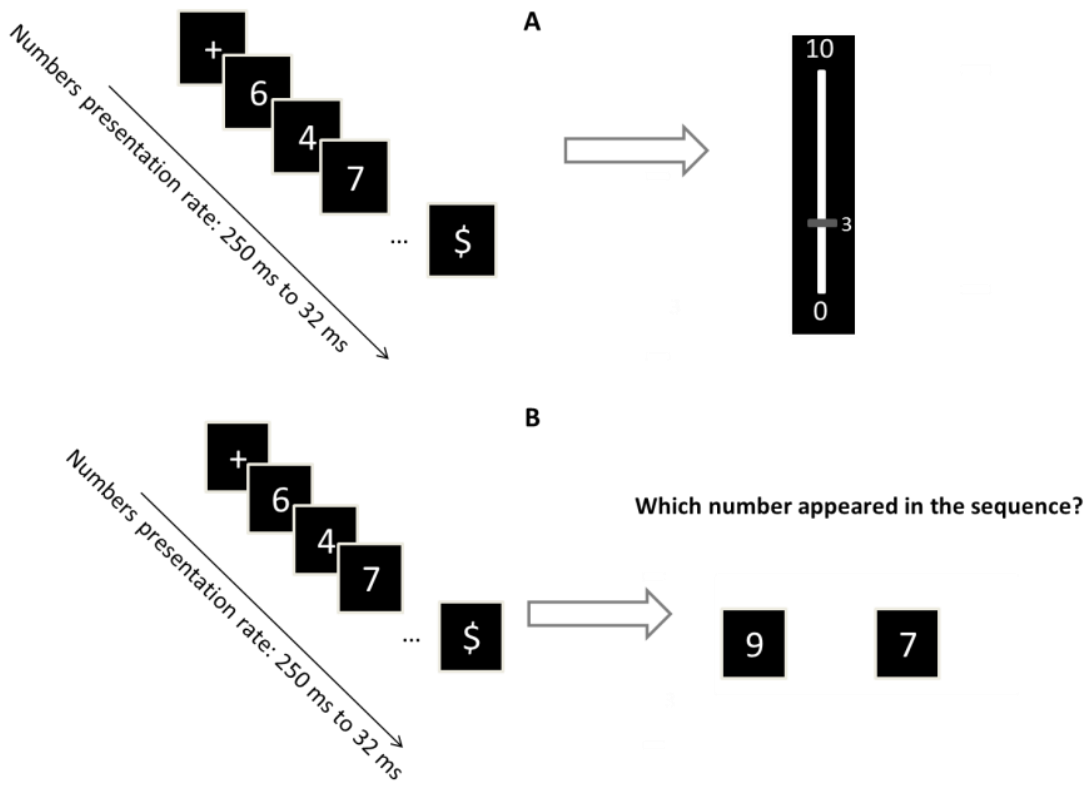

3 We sample from a combination of uniform and skewed (Fig 1C), rather than Normal or (exclusively) uniform distributions, in order to make the task too difficult for a strategy that samples few (1-2) items out of the sequence and use them to compute the average. Whereas such a strategy can succeed with sequences generated from Gaussian or uniform distribution, it will fail badly with skewed distributions, due to the presence of outliers. 


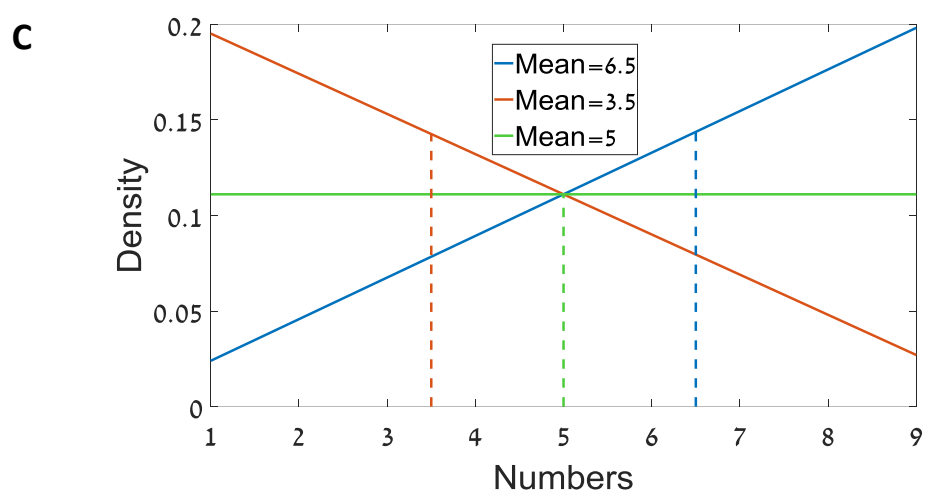

Figure 1. (A) averaging task procedure. (B) memory task procedure. C) The distributions from which the numbers were sampled; The green distribution is uniform and its average is 5; the red distribution samples lower numbers (but has occasionally large outliers) and has an average of 3.5. The Red-distribution samples more high numbers but has occasional low numbers and has an average of 6.5. In each trial, a sequence of 12 numerals randomly sampled from one of these 3 distributions is presented. This mixture of distributions increases the range of sequence-average compared to what would be obtained if the sequences would only have been sampled from a single distribution.

In Exp. 2 \& 3, after the subjects completed the averaging task at the fastest presentation rate $(32 \mathrm{~ms} /$ item $)$, they were tested on a memory task in two blocks with presentation rates of 50 and $32 \mathrm{~ms} /$ item. In this Memory task (Fig. 1B); after each sequence, the participants were presented with two probe-numbers, one which was presented in the sequence (e.g., "7" in Fig 1B) and a second which was not presented in that sequence (e.g., "9" in Fig 1B). The participants were instructed to choose the number that was presented in the last sequence (out of the two numbers and guess if unsure). In order to vary the difficulty level in this task, the probe-number which appeared in the sequence could have been a number that appeared only once in the sequence $(66.6 \%$ of the trials) or twice $(33.3 \%$ of the trials). We expected that participants will be near chance at recognizing the target as being presented in the sequence, especially for the cases in which it was presented only once.

\section{Data analysis and dependent variables}

To quantify the averaging-performance, we use the RMSD (root mean square deviation between each estimation and the actual sequence average, across all trials). The RMSD measure was normalized by dividing the actual RMSD by the RMSD that would be obtained for the same sequences based on a random response (i.e., a random selection from 1-9). ${ }^{4}$ In addition, we estimated, for each participant, the averaging

4 Based on this normalization, values smaller than 1 correspond to an averaging performance that exceeds chance. This, however, assumes that chance responses are uniformly distributed on the (1-9) interval, which is likely to be incorrect due to the 'central tendency' of judgments - a bias towards middle of the range, in the face of 
performance the subject could achieve, based on the memory performance (deviation from chance). This memory-deviation from chance allows us to estimate the number, $k$, of individual elements the subject holds in WM, at the end of a trial, and which the subject could (exclusively) rely on, to calculate (off-line) the average (without any calculation mistake). Obviously this estimation (labeled as Memory-Based Idealobserver Average; MBIA) overestimates the performance that can be achieved by offline calculating the average from remembered elements of the sequence. We predicted that the actual average will exceed the $\boldsymbol{M B I A}$ across the subjects for fastest presentation rates, indicating a gist based computation without robust access to the elements of the sequence.

\section{Experiment $1 \& 2$ results (pilots)}

Two pilot tests were conducted. In Exp. 1, 10 participants performed the averaging task only (see figure 2A, grey dashed lines from 250-50 ms/item). In Exp. 2, another 5 participants took part in both of the tasks above (averaging \& memory). Fig 2A (color lines from 250 to $32 \mathrm{~ms} /$ item) shows the averaging-performance (normalized RMSD ${ }^{4}$ ) of these 5 participants at each presentation rate (the solid black line in Fig 2A shows the average across both groups). We observe that the normalized RMSDs are all< 1 . To test for deviation from chance (see footnote-4), for each subject, we compared the RMSD in the actual trials to that from shuffled trials. We observe that even for the fastest presentation rate $(32 \mathrm{~ms} /$ item), the averaging precision $(\mathrm{Exp}-2)$ exceeds chance: Actual RMSD=.45; Shuffled RMSD=.69; $\mathrm{t}(4)=-6.2, \mathrm{p}<.01 .^{5}$

What could be the mechanism that mediates these reliable RSVP gist estimations? As numbers can be encoded in WM and used to symbolically calculate the average, we wish first to consider two such WM-based variants: an online (running average calculation) and an offline (end of sequence) one. Due to the fast rate (at 50 or $32 \mathrm{~ms}$, item) carrying out an online calculation of a running average is unlikely. ${ }^{6}$ An offline

uncertainty (Anobile et al, 2012; Hollingworth, 1910). For this reason, we do not rely on the 'normalized' RMSD value to estimate if the averaging precision exceeds chance. Instead, we compare for each subject, the RMSD of the actual trials with that of shuffled trials (which is invariant to central tendencies effects). The normalization is useful, however, in order to generate a simple meaningful value, which is not subject dependent (as the shuffled RMSD is) and which can be compared with the simulated version of WM-based estimation (see Fig. 2B).

5 In Exp.1, we observe that for the fastest presentation rate $(50 \mathrm{~ms} /$ item $)$, the averaging precision also exceeds chance: Actual RMSD=.38; Shuffled RMSD=.56; $t(9)=-8, \mathrm{p}<.001$.

6 A running average for a sequence $\left(\mathrm{x}_{1}, \ldots \mathrm{x}_{\mathrm{n}}\right)$ can be carried out exactly, by computing the running average, $y_{n}$, in a recursive way, as $y_{1}=x_{1} ; y_{2}=1 / 2 y_{1}+1 / 2 x_{2} ; y_{3}=2 / 3 y_{2}+1 / 3 x_{3}$, $\ldots$ with weights that decreases along the sequence. (Note that, an alternative and more simple, leaky integration with a constant weight would generate a strong recency bias, which is not found in the data). Previous studies of numerical averaging (at slower rates of 
calculation, based on few samples that have been maintained in WM, could however, contribute to the averaging performance. To estimate if the actual performance can be accounted for via such an WM-based symbolic calculation, we show in Fig 2B, a simulation that depicts the averaging-RMSD (same normalization) that could be achieved if a subject used $k$-samples out of the sequence from memory without any error in the calculation of the average from those $k$-samples. We see that to achieve the actual (average) level of performance (in the $32 \mathrm{~ms}$ condition), a subject would need to remember and use on average (without distortion) more than two samples out of the sequence (i.e., 2.4; see black line corresponding to the group average in Fig 2A and its mapping onto Fig 2B). As we show below (Fig. 2D), this overestimates the actual memory performance ( $k$-parameter of each subject), which (at those fast rates) is much lower (dotted black line in fig 2D represents the average $k$-parameter across participants=.89).

A

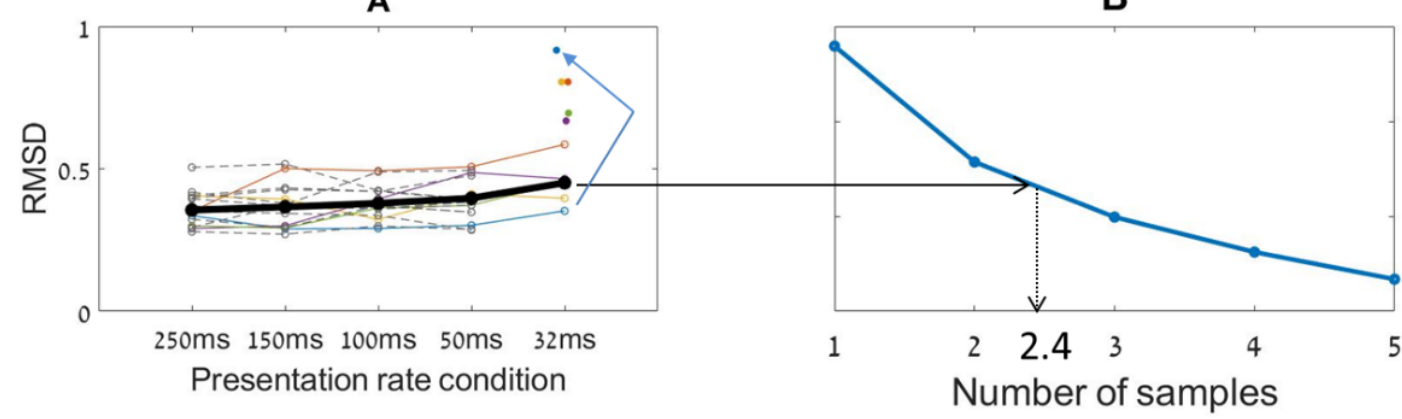

C

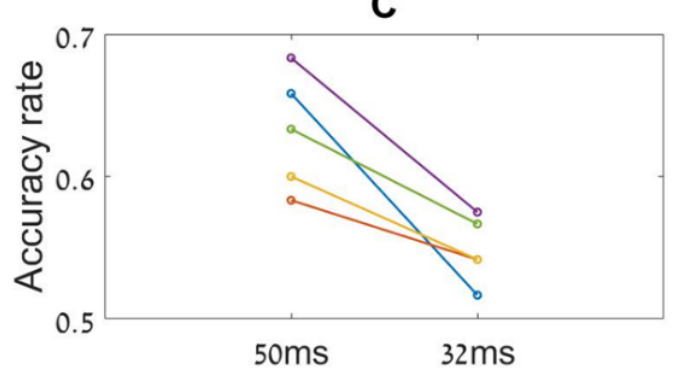

D

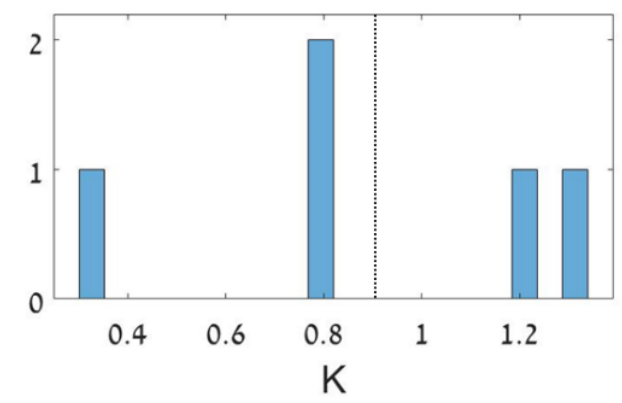

Figure 2. (A) Subjects' averaging performance measured in Root mean square deviation for each presentation rate (black line represents the average RMSD, grey dashed lines represent participants from pilot 1 and colored lines represent participants from pilot 2) and the MBIA performance estimated from the memory task accuracy rate of each subject (from pilot 2) in the $32 \mathrm{~ms}$ condition (dots); note that for each subject, the colored dots are higher than the corresponding colored-line (the difference between the averaging performance and MBIA is illustrated for a

$2 / \mathrm{sec}$ ) have already provided evidence against such a mechanism (Malmi \& Samson, 1983). At rates faster than $10 / \mathrm{sec}$, such an estimation becomes even more unlikely so we do not consider it further. 
representative subject (blue arrows). (B) Averaging simulation in which $\mathrm{k}$ items are sampled and are accurately averaged. (C) Memory task accuracy for each presentation rate. (D) The distribution of k-parameter, for the $32 \mathrm{~ms}$ condition, based on the memory task accuracy rate. The black dashed line represents the average $k$ parameter across participants (.89).

To estimate the number of items that each participant has access to (at the end of a trial) out of the twelve presented on the fastest (32 ms/item and $50 \mathrm{~ms} / \mathrm{item}$ ) RSVP conditions, we examined the memory performance for RSVP sequences with the same presentation rate, which are carried out after the averaging task. The rationale is that, if the way in which the participants carried out the averaging estimation was to (offline) calculate the average based on the few samples they could remember at the end of the sequence, it should be even easier for them to just recognize which of two memory probes (one presented in the sequence and the other not) was part of the sequence. If one of the two probes matches any of the $k$-samples held in WM the participant should select it, and if neither does, the participant should guess ${ }^{7}$. Fig $2 \mathrm{C}$ shows the memory performance at the two fastest presentation rates (50 and 32 $\mathrm{ms} /$ item) for the 5 subjects in Exp-2. We observe the memory performance decreases with presentation rate, approaching chance level $(50 \%)$ at the faster rate $(32 \mathrm{~ms})$; see Supplement (Fig S4) for dependency of the memory performance with repetition of the probed item. For each subject, based on her/his deviation from chance in the 32 ms condition, we extracted the $k$-value (estimate of the number of samples out of 12 that the subject can recognize at the end of the sequence). The distribution of k-values is plotted in Fig 2D, indicating that most subjects remember (or have access to) less than 1 item per sequence (mean $k=.89$ ). For each subject in Exp-2, we use this $k$ estimation to compute an $\boldsymbol{M B I A}$ (memory-based ideal observer average) estimation of the RMSD (based on the simulation in Fig 2B). These RMSD estimates are presented as colored dots, for each participant in Fig $2 \mathrm{~A}$ at the $32 \mathrm{~ms}$ condition. We observe that for each the 5 participants, the $\boldsymbol{M B I A}$ accuracy is lower (RMSD is higher) than the actual one at the $32 \mathrm{~ms}$ rate (Fig 2A; see blue arrows for illustration of one subject). This difference is highly significant for the group $(t(4)=4.6, p<.001)$.

7 This results in an estimation of $k$ based on $M=k / 12+(1-k / 12) * .5$; where $M$ is the memory performance and $k$ is the number of items held in WM (for $\mathrm{k}=0, \mathrm{M}=.5$ ). Since in our experiment we also have $1 / 3$ of trials, in which the probe corresponds to an item that was presented twice, we relied instead on a computer simulation, which generated the items and the memory probes, exactly as in the experiment, and randomly samples $K$ items out of the sequence to be maintained in WM (if an item was repeated in the sequence it has a higher probability to be sampled). It then provided a correct response if the probe matches one of those items, and guesses (with $\mathrm{p}=.5$ ) otherwise. As the memory performance changes in steps with the k-variable (which is an integer), we extracted $k$ from memory performance by interpolation; Matlab simulation code is available from the authors. A non-integer $k$-value, such as $k=1.5$ corresponds to a situation in which the number of items encoded in WM is 1 or 2 , each in one half of the trials. 


\section{EXPERIMENT 3}

This is a pre-registered replication experiment based on the results from Exp. 2 (https://osf.io/7cj9g/). The aim was to demonstrate that at high RSVP rates (50 and 32 ms/item) participants are able to make gist (average) estimation with a precision that exceeds what they can achieve based on memory of individual items at the end of a sequence (MBIA estimate). A power analysis (based on Exp-2) indicated that the sample size needed for examining the difference in real averaging performance and MBIA for each subject in the $32 \mathrm{~ms}$ condition at a power of $99 \%$ is 13 participants ( $\mathrm{P}$ value<.001). We recruited and tested two extra participants (a total of 15) in case there would be a problem with some of the participants' data (or with cancellations).

\section{Experiment 3 results}

15 participants took part in Exp. 3. The experiment procedure was exactly the same as described for Exp. 2. Fig 3A shows the mean averaging-performance (normalized RMSD) averaged over all subjects at each presentation rate. Again, for each subject, the RMSD in the actual trials was compared to that from shuffled trials and we observed that even for the fastest presentation rate $(32 \mathrm{~ms} /$ item $)$, the averaging precision exceeds chance: Actual $\mathrm{RMSD}=.52$; Shuffled $\mathrm{RMSD}=.67 ; \mathrm{t}(14)=-4.7$, $\mathrm{p}<.001$. In Fig 3B, we present a simulation that shows the averaging-RMSD (same normalization) that could be achieved if the subject used k-samples out of the sequence from memory (without any error). We see that to achieve the actual level of performance, the subjects needed to remember and use (without distortion or calculation error) 2 or more samples out of the sequence.

Fig 3C shows the mean memory performance for the two conditions (item duration of 50 and $32 \mathrm{~ms}$; see Supplement for the memory performance as a function of the serial position in the list). We observe that the memory performance decreases with presentation rate (although this trend did not reach significance, $t(14)=1.7, p=.09$ ), approaching (but exceeding) chance level $(50 \%)$ at the faster rate $(32 \mathrm{~ms})$, for the group. For each subject, based on her/his deviation from chance, in each condition, we extracted the $k$-value (estimate of the number of samples out of 12 that the subject can recognize at the end of the sequence). The distribution of k-values in the $32 \mathrm{~ms}$ condition is plotted in Fig 3D, indicating that most subjects remember (or have access to) less than 2 numbers per sequence (mean k-parameter=.98; see black dotted line). For each subject, we use this $k$-estimation to compute an $M B I A$ estimation of the averaging-RMSD for each presentation-rate condition $(32 / 50 \mathrm{~ms} / \mathrm{item})$. These averaged RMSD estimates are presented in Fig 3A (blue line) and the actual vs. MBIA averaging precision for (individual participants) are shown in Fig 4A-B. We observe that in both conditions ( $32 \mathrm{~ms} /$ item \& $50 \mathrm{~ms} /$ item), the $M B I A$ accuracy is lower (RMSD higher) than the actual subjects' accuracy (32 ms/item: $t(14)=4.58, p<.001,50$ ms/item: $t(14)=5.3, p<.001$ ), replicating the results from Exp-2. 
A

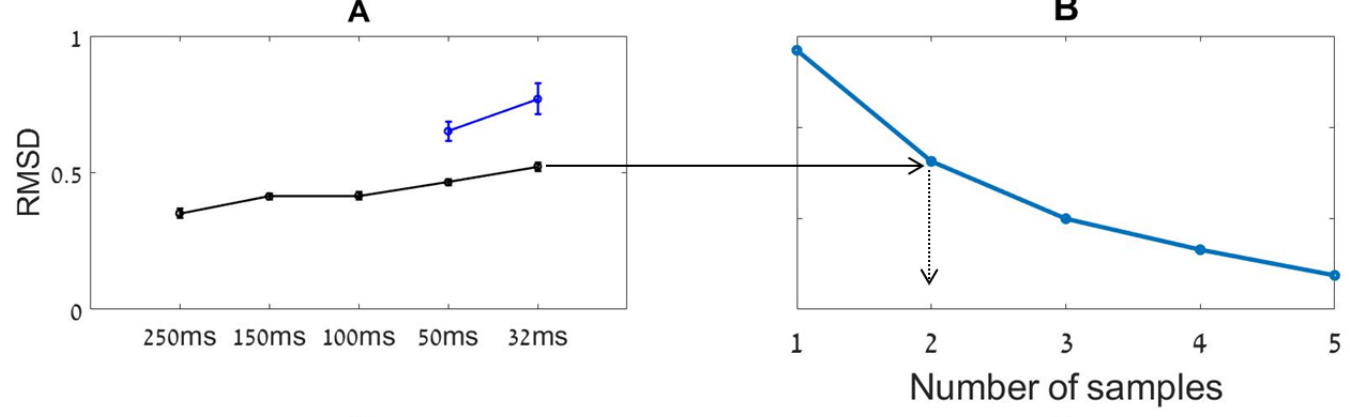

C

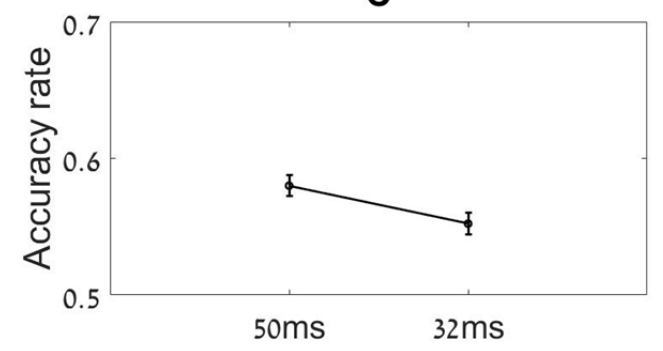

D

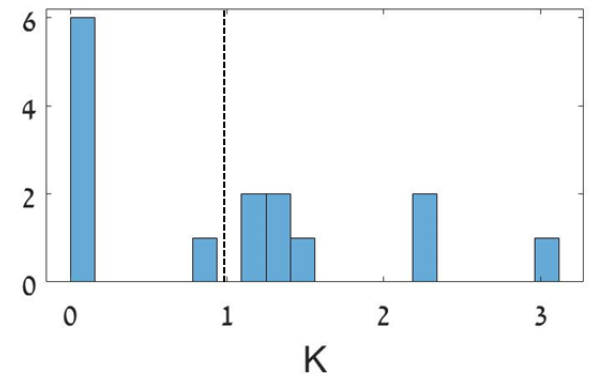

Figure 3. (A) Subjects' averaging mean performance measured in RMSD for each presentation rate (black line) and the mean MBIA performance estimated from the memory task accuracy rate of each subject in the $50 \mathrm{~ms}$ and $32 \mathrm{~ms}$ conditions (blue line). Error bars correspond to SEM (Cousineau, 2005); these are very small for the RMSD line. (B) Averaging simulation in which kitems are sampled and are accurately averaged. (C) Memory task mean accuracy rate for each presentation rate. Error bars correspond to SEM. (D) The distribution of k-parameter for the $32 \mathrm{~ms}$ condition, based upon the memory task accuracy rate. The black dashed line represents the average $k$-parameter across participants (.98). 
A

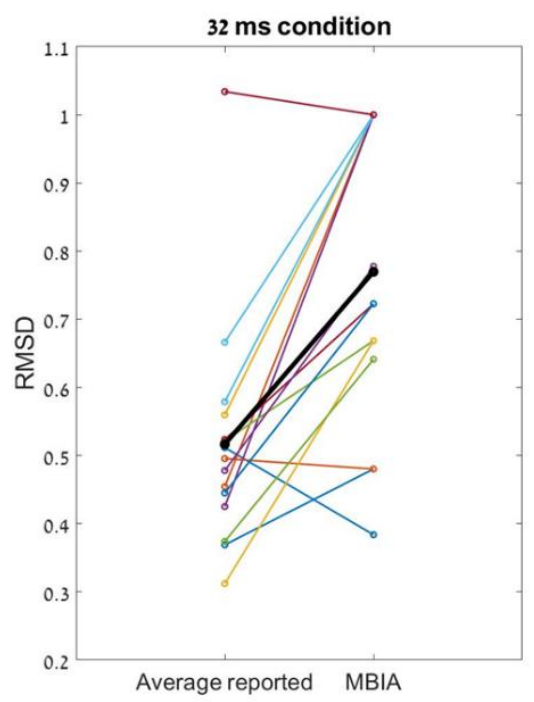

B

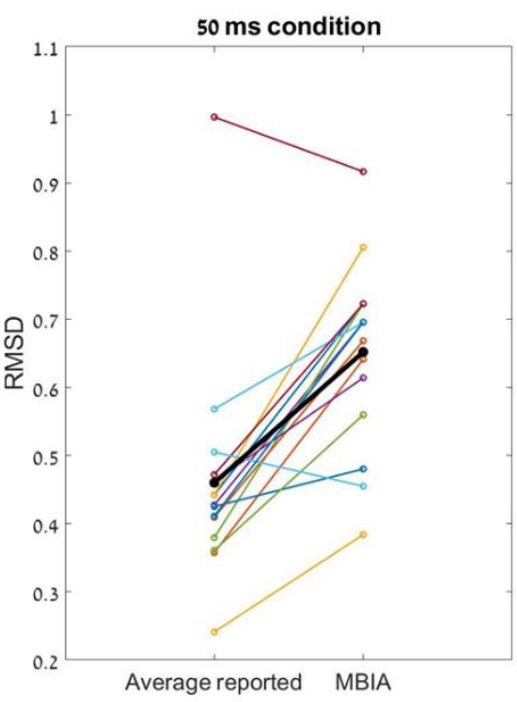

C

$\beta=0.1, \quad p<.001$

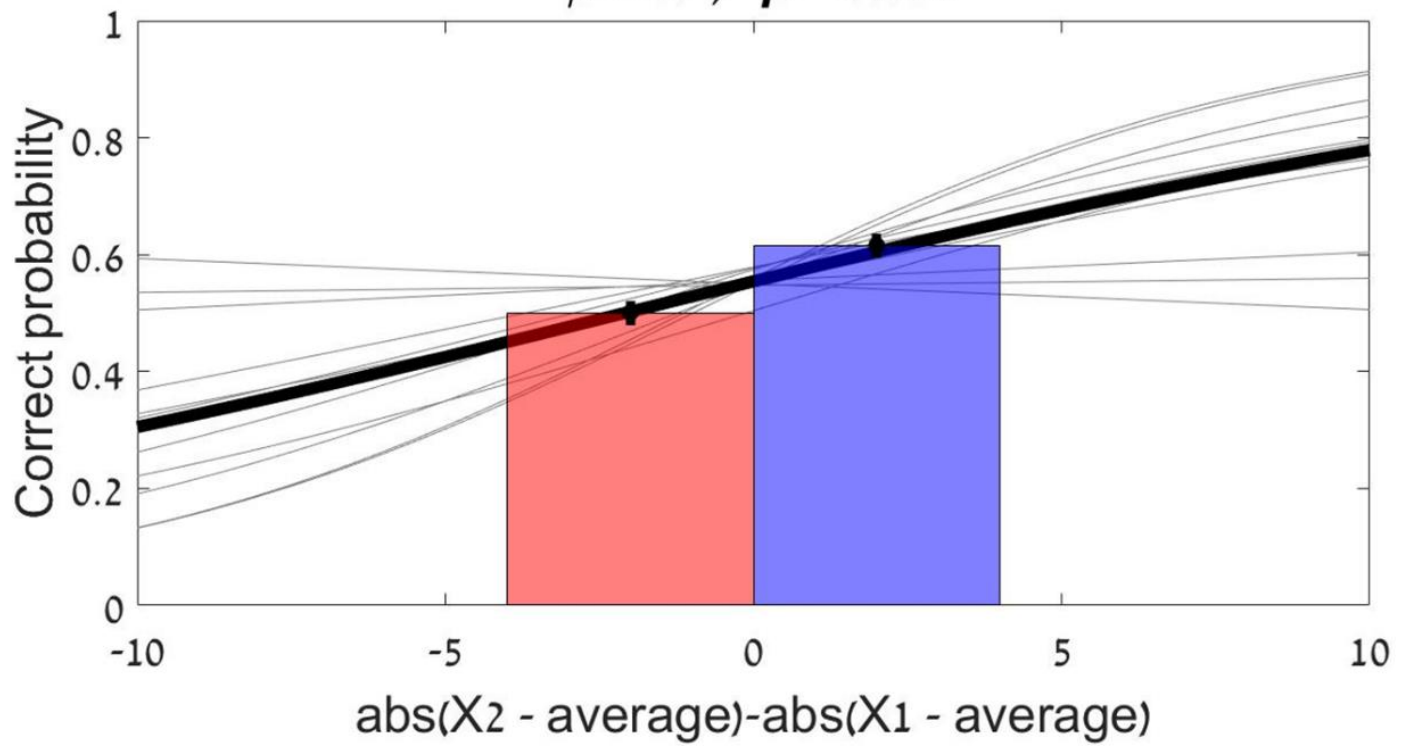

Figure 4. A-B) Subjects' real averaging performance and MBIA performance measured in RMSD for the $32 \mathrm{~ms}$ condition (left) and the $50 \mathrm{~ms}$ condition (right). Each line represents a subject (black line represents the average across all participants). In both presentation-rate conditions, at least 12 out of the 15 subjects have a positive slope, showing that their actual performance exceeded their MBIA performance. C) A mixed-effect logistic regression on memory performance (y-axis) with the difference in distance of the sequence-present probe (X1) and the sequenceabsent probe (X2) from the (task irrelevant) sequence-average, Abs (X2-Average) Abs (X1 -Average) as a predictor, and with random intercepts and slopes at the participant level. Solid curve corresponds to the group and gray curves to individual participants. Memory accuracy increases when the sequence-present probe is closer to the sequence-average than the sequence-absent probe. In addition, Panel C shows memory performance, for two type of trials: i) sequence-absent probe is closer to the 
sequence's average (red bar) and ii) the sequence-present probe is closer to the sequence's average (blue bar).

The results indicate that the averaging mechanism is not based on the encoding of sequence samples in WM. We now wish to examine the opposite direction of influence. Is there an automatic estimation of the average taking place (although being task irrelevant and resulting in a bias) during the memory task (Khaiat \& Hochstein, 2018) To asses this, we performed a mixed-effect logistic regression on the memory performance with the difference between the distances of the matching and the nonmatching probes from the sequence average as the predictor and with random intercepts and slopes at the participant level. As illustrated in Fig 4C, the memory performance increases when the sequence-present probe is closer to the (task irrelevant) sequence-average than the sequence-absent probe, and decreases when the non-matching probe is closer to the sequence-average. This indicates that (average) gist extraction is automatic, as it takes place at high rates and even when it is task irrelevant, leading to a bias in the relevant (memory) task (see Khaiat \& Hochstein, 2018).

\section{Discussion}

In three experiments (including a total of 30 participants), we examined the ability of human observers to estimate the average of RSVP numerical sequences. We found that this ability was impressive (compared to chance based responding) for rates as fast as 32 $\mathrm{ms} /$ item, for which one can "barely see" the individual numbers in the sequence ${ }^{8}$. In our second and third experiments, we examined, in addition to averaging estimation, the memory identification for a single probe-item presented in the sequence. For the fastest presentation rates (50 or $32 \mathrm{~ms} / \mathrm{item}$ ), this memory performance is low (at all temporal positions within the sequence), but slightly exceeds chance (see Fig S2, in Supplement), indicating that the numbers are visible (to some extent), but that most of them were not encoded in WM (see Fig S1 in Supplement, showing no recency). In Experiments 2 and 3 , we used the deviation from chance in the memory task to estimate how many numbers ( $k$ ) from an RSVP sequence each subject maintained in WM at each presentation rate. Those $k$-estimates were low (Fig 2-3D) and for most participants, they did not exceed a single-item ( $k$-values in Fig 2D-3D). Based on these estimates we computed, for each participant, a memory based ideal observer measure of averaging (MBIA). The MBIA is the RMSD precision that a participant would attain, based on an (error-less), offline calculation from the numbers encoded in WM, at the end of the RSVP. For most of the participants ( $83 \%$ ) and in both experiments (2 and 3), this MBIA measure had a reduced precision compared to the participant's performance (see Fig 4). Furthermore, we found that, rather than the averaging estimation being memory based, the memory performance

8 Informal subjective reports indicate that at the highest rates (50 and $32 \mathrm{~ms} / \mathrm{item})$ the subjects perceives only few of the numbers in a sequence in a fleeting way. These informal reports are consistent with memory performance on the last sequence-item (see below and Fig S2 in the Supplement). 
is biased by the (task irrelevant) distance of the memory probes from the sequenceaverage (Fig $4 \mathrm{c}$ ), indicating that this gist estimation is automatic and takes precedence.

The results provide further support for the idea that an intuitive estimation of the average (a type of gist extraction) is online and, automatically carried out (see also Khayat \& Hochstein, 2018), even for high rate RSVP sequences, in which the items are barely visible. One possible mechanism accounting for these estimations involves the online activation of numerosity detectors in the parietal cortex (see Brezis et al, 2017, for support from a brain stimulation study), and the reliance on a population code mechanism that accumulates within each sequence (see illustration in Fig 5) and which extracts the average from the population response after each trial, accounting for a precision that increases with the length of the list due to the averaging of encoding noise (see Fig 5; adapted from Brezis et al., 2018). The novelty of our results is to show that such a process can operate at high RSVP rates and that the average extraction mechanism exceeds actual WM capacity and that takes precedence on memory reports.
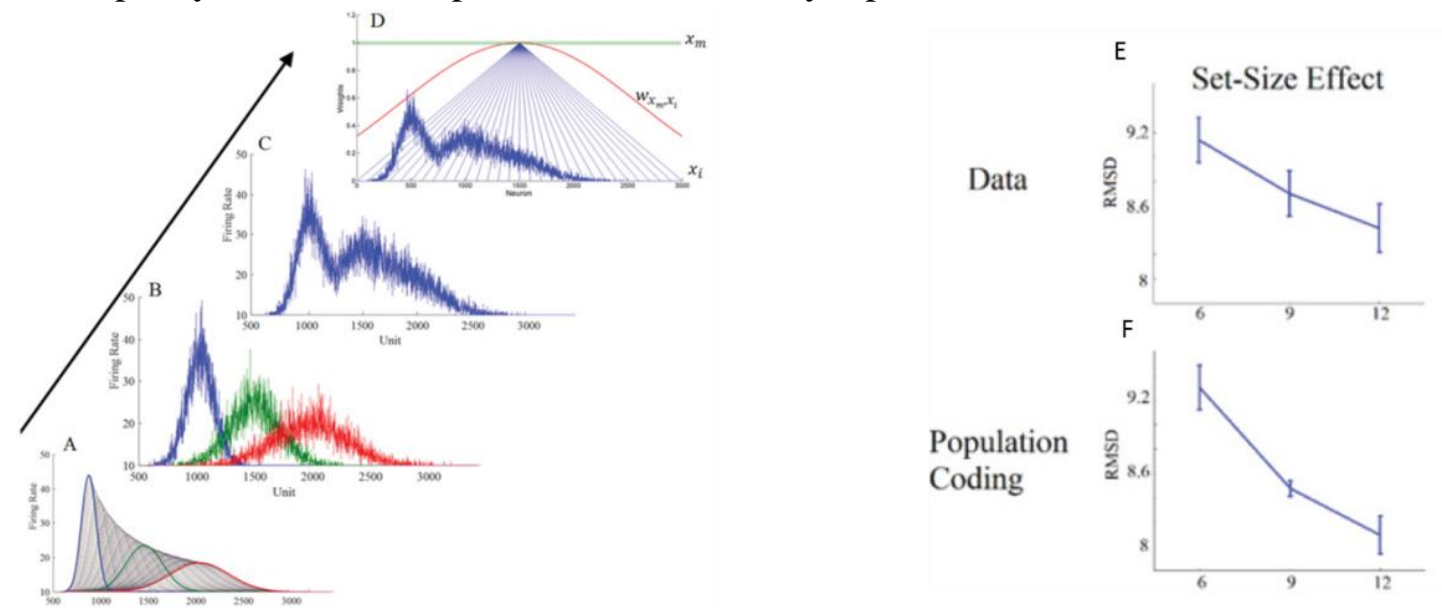

Fig 5. A population-code model of numerical averaging (Brezis et al, 2017). The model assumes that symbolic numbers activate analog numerosity detectors in the parietal cortex that are broadly tuned to numerical magnitudes (panel-A, bottom left). This is illustrated for an arbitrary sequence of 3 numbers (red, green, blue) population responses (panel-B), which are accumulated (panel-C) into a population profile that represents the sequence (panel-C). The center of mass of this population response is decoded corresponding to the estimated average (panel-D). The model accounts for an improvement of the average precision (RMSD) with the length of the sequence (right panels showing model and experimental data).Adapted from Brezis et al, (2018); Neural Computation.

One alternative explanation of the results is that our memory capacity- $k$ underestimates the actual number of values encoded by the subjects (which could be higher than our kestimate), if those numbers are encoded with some encoding noise (Bays \& Husain, 2008) ${ }^{9}$. Note that a similar objection can be raised towards the statement that summary

9 Such encoding noise would reduce the memory performance but could average out (across say, four items) in averaging. 
statistics are carried out without the encoding of individual items in array studies (Ariely et al., 2001), as small encoding noise could specifically reduce the performance in the single items tests but would average out in the average estimate. Unlike in most studies of summary statistics, however, here we used sequences of symbolic numbers, which in the case of conscious perception are minimizing encoding noise ${ }^{10}$. Finally, one may consider the possibility that the samples held in WM are not random, but rather selected in a more sophisticated way (say the minimum and the maximum of the sequence) resulting an enhanced precision (Myczek \& Simons, 2008). This hypothesis, however, is not supported by the memory data, which show no difference as a function of the memory probe (see Fig S2).

These results are also interesting in relation to the debate about the conscious experience of items in RSVP. The presentation rate we used here, exceeded those employed in most RSVP studies (of around $100 \mathrm{~ms} / \mathrm{item}$ ). At these faster rates (50 or $32 \mathrm{~ms} / \mathrm{item}$ ), we observe that participants' memory recognition of individual items is close (but slightly exceeds) chance (Fig S1 in Supplement; see Potter, 1976 for similar results). The lack of serial position effect in this data, suggest that at this fast rate, most of the items are not encoded in WM (an encoding followed by forgetting hypothesis would predict recency, which is not supported by the data). There are two possibilities, however, we can consider about the conscious experience of the items. The first one, is that the items are subject to a fleeting conscious experience ( $\mathrm{Su}$, Barnard \& Howard, 2011), that allows the extraction of gist to proceed, while the second is that the gist extraction is carried out subliminally (Van Opstal, de Lange \& Dehaene, 2011; Bowman, Filetti, Janssen, Su, Alsufyani, \& Wyble,2013). The latter hypothesis is supported by the lack of a correlation (across participants) between the memory performance on the last memory item (which could provide a measure of the items' visibility) and the RMSD averaging performance (see Fig S3 in the Supplement). Whereas, future experiments will be needed to conclusively distinguish between these alternatives, our results indicate that gist processing can take place from RSVP sequences (Luck, Vogel \& Shapiro, 1996), in the absence of a robust (WM) access to the elements that could be used to make those estimations.

\section{References}

Anobile, G., Cicchini, G. M., \& Burr, D. C. (2012). Linear mapping of numbers onto space requires attention. Cognition, 122(3), 454-459.

10 From a perceptual perspective, any two digits are equally similar (e.g., 4 vs 5 or 4 vs 9).

So there is little sense to a precision encoding parameter as with analog visual properties. It is possible that symbolic numbers, activate non-symbolic analog representations of numerosity (Dehaene et al), but this is exactly what our population-coding account (Brezis et al., 2018) proposes. 
Ariely, D. (2001). Seeing sets: Representation by statistical properties. Psychological science, 12(2), 157-162.

Baek, J., \& Chong, S. C. (2020). Distributed attention model of perceptual averaging. Attention, Perception, \& Psychophysics, 82(1), 63- 79.

Block, N. (2011). Perceptual consciousness overflows cognitive access. Trends in cognitive sciences, 15(12), 567-575.

Bowman, H., Filetti, M., Janssen, D., Su, L., Alsufyani, A., \& Wyble, B. (2013). Subliminal salience search illustrated: EEG identity and deception detection on the fringe of awareness. PLoS One, 8(1), e54258.

Brezis, N., Bronfman, Z. Z., \& Usher, M. (2015). Adaptive spontaneous transitions between two mechanisms of numerical averaging. Scientific reports, 5(1), 1-11.

Brezis, N., Bronfman, Z. Z., \& Usher, M. (2018). A perceptual-like population-coding mechanism of approximate numerical averaging. Neural Computation, 30(2), 428446.

Bronfman, Z. Z., Jacobson, H., \& Usher, M. (2019). Impoverished or rich consciousness outside attentional focus: Recent data tip the balance for Overflow. Mind \& Language, 34(4), 423-444.

Chong, S. C., \& Treisman, A. (2003). Representation of statistical properties. Vision research, 43(4), 393-404.

Chong, S. C., \& Treisman, A. (2005). Statistical processing: Computing the average size in perceptual groups. Vision research, 45(7), 891-900.

Cousineau, D. (2005). Confidence intervals in within-subject designs: A simpler solution to Loftus and Masson's method. Tutorials in quantitative methods for psychology, 1(1), 42-45.

Dakin, S. C. (2001). Information limit on the spatial integration of local orientation signals. JOSA A, 18(5), 1016-1026.

Dehaene, S., Kerszberg, M., \& Changeux, J. P. (1998). A neuronal model of a global workspace in effortful cognitive tasks. Proceedings of the national Academy of Sciences, 95(24), 14529-14534.

Dehaene, S., \& Naccache, L. (2001). Towards a cognitive neuroscience of consciousness: basic evidence and a workspace framework. Cognition, 79(1-2), 1-37. 
Haberman, J., Harp, T., \& Whitney, D. (2009). Averaging facial expression over time. Journal of vision, 9(11), 1-1.

Haberman, J., \& Whitney, D. (2011). Efficient summary statistical representation when change localization fails. Psychonomic Bulletin \& Review, 18(5), 855-859.

Hollingworth, H. L. (1910). The central tendency of judgment. The Journal of Philosophy, Psychology and Scientific Methods, 7(17), 461-469.

Khayat, N., \& Hochstein, S. (2018). Perceiving set mean and range: Automaticity and precision. Journal of vision, 18(9), 23-23.

Kouider, S., Sackur, J., \& de Gardelle, V. (2012). Do we still need phenomenal consciousness? Comment on Block. Trends in cognitive sciences, 16(3), 140.

Lee, K. R., Dague, T. D., Sobel, K. V., Paternoster, N. J., \& Puri, A. M. (2021). Set size and ensemble perception of numerical value. Attention, Perception, \& Psychophysics, 1-10.

Luck, S., Vogel, E. The capacity of visual working memory for features and conjunctions. Nature 390, 279-281 (1997).

Luck, S. J., Vogel, E. K., \& Shapiro, K. L. (1996). Word meanings can be accessed but not reported during the attentional blink. Nature, 383(6601), 616-618.

Malmi, R. A., \& Samson, D. J. (1983). Intuitive averaging of categorized numerical stimuli. Journal of Verbal Learning and Verbal Behavior, 22(5), 547-559.

Myczek, K., \& Simons, D. J. (2008). Better than average: Alternatives to statistical summary representations for rapid judgments of average size. Perception \& psychophysics, 70(5), 772-788.

Parkes, L., Lund, J., Angelucci, A., Solomon, J. A., \& Morgan, M. (2001). Compulsory averaging of crowded orientation signals in human vision. Nature neuroscience, 4(7), 739-744.

Potter, M. C. (1976). Short-term conceptual memory for pictures. Journal of experimental psychology: human learning and memory, 2(5), 509.

Robitaille, N., \& Harris, I. M. (2011). When more is less: Extraction of summary statistics benefits from larger sets. Journal of vision, 11(12), 18-18. 
Rosenbaum, D., de Gardelle, V., \& Usher, M. (2021). Ensemble perception: Extracting the average of perceptual versus numerical stimuli. Attention, Perception, \& Psychophysics, 1-14.

Rosenbaum, D., Glickman, M., \& Usher, M. (2020). Extracting summary statistics of rapid numerical sequences.

Sperling, G. (1960). The information available in brief visual presentations. Psychological monographs: General and applied, 74(11), 1.

Su, L., Bowman, H., \& Barnard, P. (2011). Glancing and then looking: on the role of body, affect, and meaning in cognitive control. Frontiers in psychology, 2, 348.

Van Opstal, F., de Lange, F. P., \& Dehaene, S. (2011). Rapid parallel semantic processing of numbers without awareness. Cognition, 120(1), 136-147.

\section{Supplement}

Memory performance in Exp-3, as a function of serial order in the list:
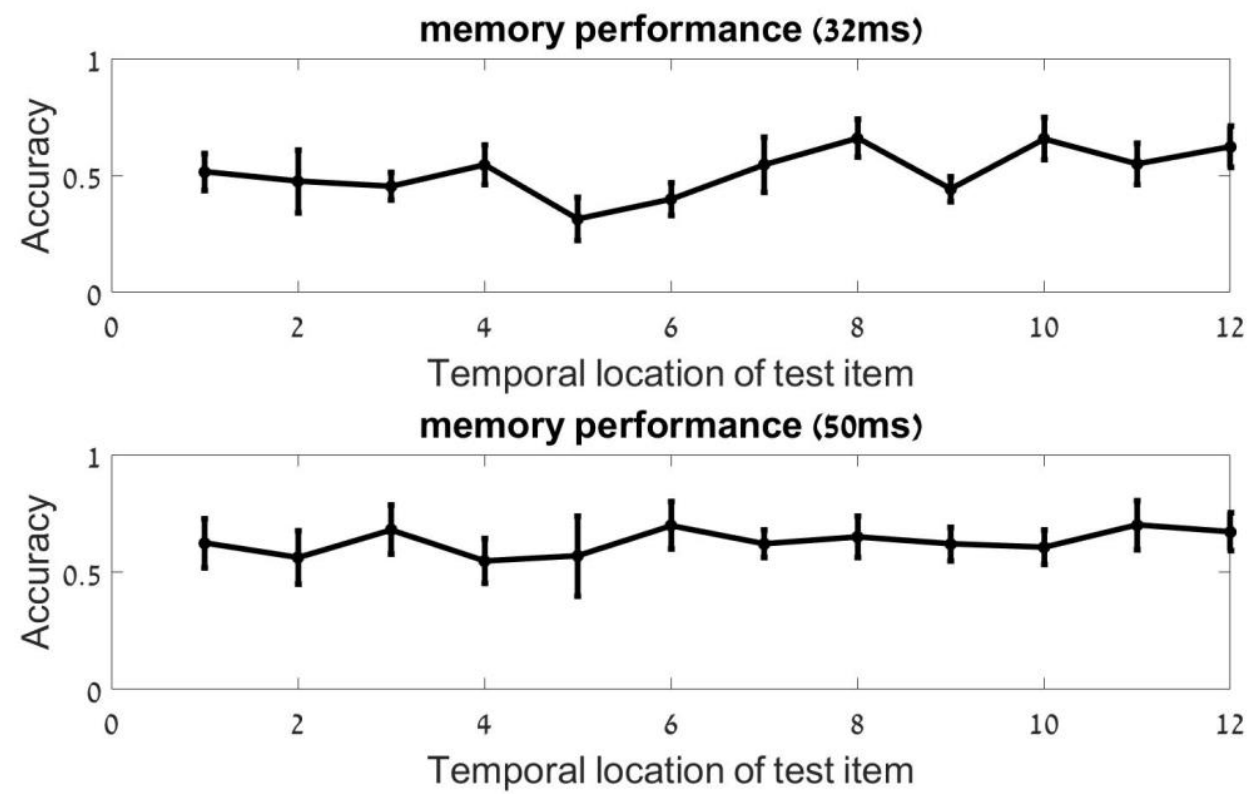
Fig S1. 2AFC memory performance for the data in Exp. 3, at the two fastest presentation rates. The performance slightly exceeds chance, but there are no serial; order effects (i.e., no recency).

Memory performance in Exp-3, as a function of tested item value:
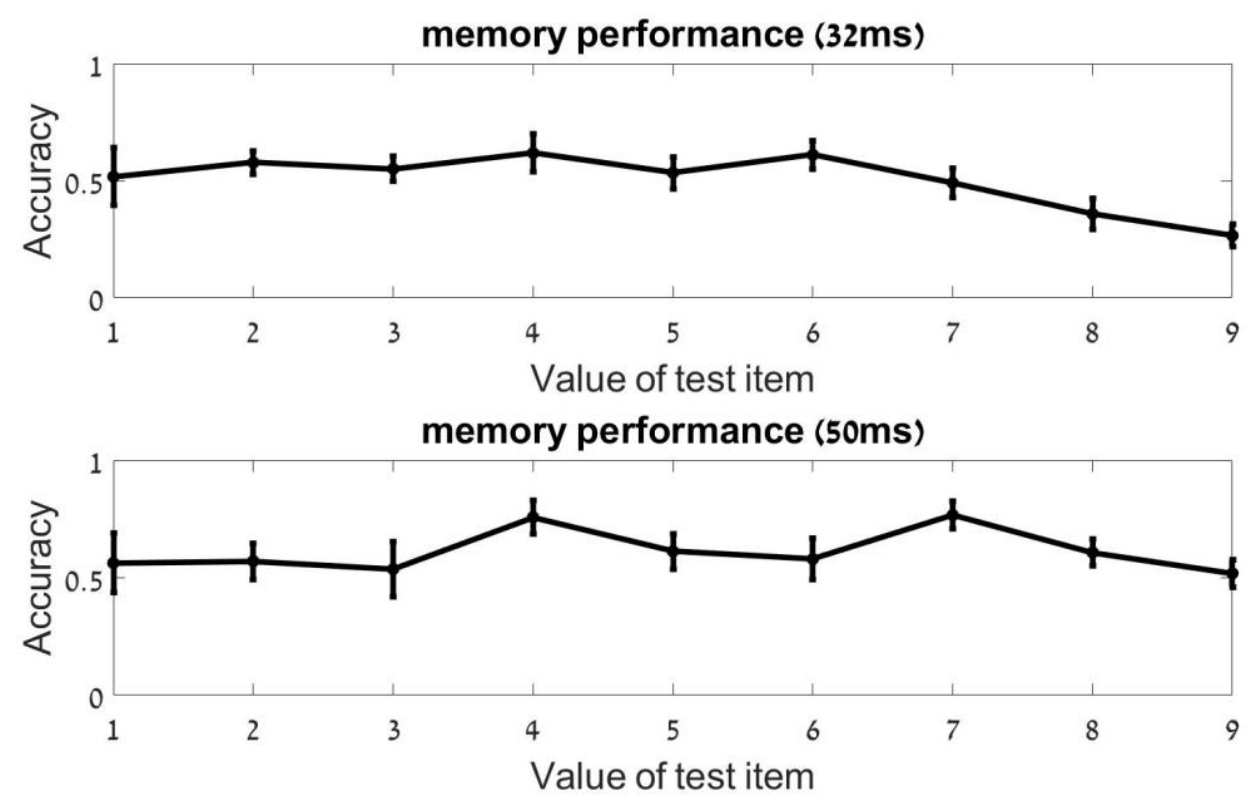

Fig S2. 2AFC memory performance for the data in Exp. 3, at the two fastest presentation rates. The performance slightly exceeds chance, but there are no value effects (i.e., no tendencies of remembering better high or low numbers). 

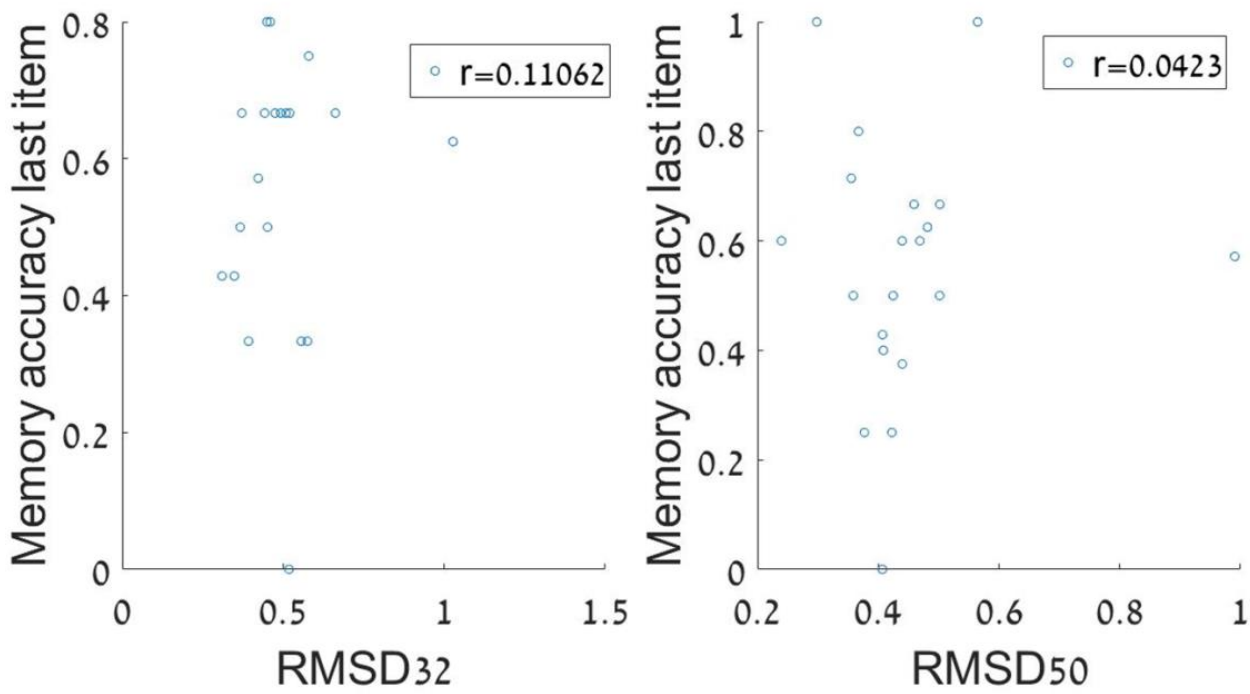

Figure S3. Correlation between accuracy in memory performance on trials in which tested item was presented last in the sequence, and averaging performance (RMSD) in the $32 \mathrm{~ms}$ condition (left panel) and the $50 \mathrm{~ms}$ condition (right panel).

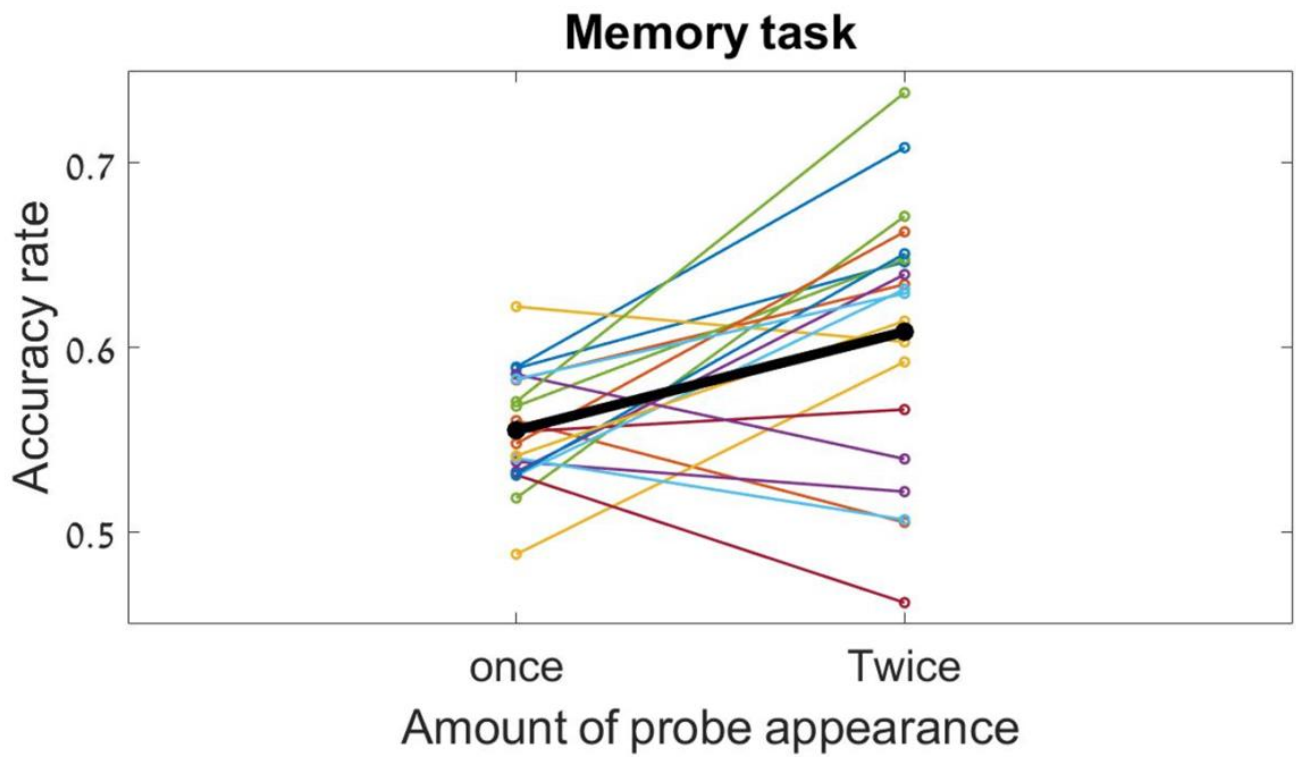

Figure S4. Memory performance as a function of difficulty level (Once= trials in which the probed item appeared once. Twice $=$ trials in which the probed item appeared twice) for each participant (colored lines) and the averaged performance (black line). A t-test revealed a significant difference in performance between difficulty levels $(t(14)=2.55, p<.01)$ showing that performance was better in trials in which the probed item appeared twice compared to once. 